\title{
Comparação entre Raquianestesia, Bloqueio Combinado Raqui-Peridural e Raquianestesia Contínua para Cirurgias de Quadril em Pacientes Idosos. Estudo Retrospectivo *
}

\author{
Comparison between Spinal, Combined Spinal-Epidural and \\ Continuous Spinal Anesthesias for Hip Surgeries in Elderly Patients. \\ A Retrospective Study
}

Luiz Eduardo Imbelloni, TSA $^{1}$, Lúcia Beato ${ }^{1}$

\begin{abstract}
RESUMO
Imbelloni LE, Beato L - Comparação entre Raquianestesia, Bloqueio Combinado Raqui-Peridural e Raquianestesia Contínua para Cirurgias de Quadril em Pacientes Idosos. Estudo Retrospectivo
\end{abstract}

\begin{abstract}
Justificativa e Objetivos - Diversas questões envolvem os estudos, as análises e o tamanho da amostra para que sejam demonstrados os benefícios da anestesia regional. Análise de dados geralmente custa menos e requer menos tempo quando comparado com amplo estudo aleatório controlado. Esta análise retrospectiva compara a raquianestesia contínua, o bloqueio combinado raqui-peridural e a raquianestesia simples para cirurgias de quadril em pacientes idosos durante quatro anos, para determinar as possíveis vantagens e desvantagens das três técnicas.
\end{abstract}

Método - Foram avaliados 300 prontuários sendo que: 100 pacientes receberam raquianestesia simples (Grupo 1), 100 receberam bloqueio combinado raqui-peridural (Grupo 2) e 100 receberam raquianestesia contínua (Grupo 3) nos últimos quatro anos. Todos os bloqueios foram realizados em decúbito lateral esquerdo. Foram avaliados: sucesso de punção, nível da analgesia, bloqueio motor de membros inferiores, qualidade da anestesia, necessidade de complementação, incidência de falhas, parestesias, cefaléia pós-punção, alterações cardiovasculares, confusão mental e delírio, transfusão sangüínea e mortalidade.

Resultados - Não existiu diferença significativa entre os grupos em relação a idade, peso e sexo. Os pacientes do grupo 2 foram menores do que os do grupo 1 e 3 . As doses utilizadas foram de 15,30 mg de bupivacaína no grupo 1; 23,68 mg no grupo 2 e 10,10 mg no grupo 3. Não foi encontrada diferença significativa $(p<0,01)$ entre os grupos, sendo menor com a raquianestesia contínua e maior com o BCRP. Existiu uma diferença significativa $(p<0,01)$ na dispersão cefálica entre os grupos 1 e 2, 1 e 3 e 2 e 3, sendo menor com a raquianestesia contínua e maior com o BCRP. Todos os pacientes apresentaram bloqueio motor completo. Não existiu diferença significativa em relação a hipotensão arterial, bradicardia,

\footnotetext{
* Recebido da (Received from) Casa de Saúde Santa Maria e Clínica São Bernardo, Rio de Janeiro, RJ.

1. Anestesiologista da Casa de Saúde Santa Maria e Clínica São Bernardo
}

Apresentado (Submitted) em 13 de agosto de 2001

Aceito (Accepted) para publicação em 01 de novembro de 2001

Correspondencia para (Mail to):

Dr. Luiz Eduardo Imbelloni

Av. Epitácio Pessoa, 2356/203 - Lagoa

22471-000 Rio de Janeiro, RJ

E-mail:imbelloni@openlink.com.br

(C) Sociedade Brasileira de Anestesiologia, 2002 parestesia e necessidade de sangue. Dezenove pacientes apresentaram confusão mental no pós-operatório, sem diferença entre as técnicas utilizadas. Não há diferença significativa na incidência de óbitos na primeira semana e no primeiro mês de pós-operatório.

Conclusões - Os estudos retrospectivos geralmente custam pouco e necessitam de menor tempo quando comparado com estudos controlados. Este estudo retrospectivo mostra que as técnicas de anestesia regional cursam com uma baixa incidência de mortalidade no primeiro mês e baixa incidência de complicações.

UNITERMOS - TÉCNICAS ANESTÉSICAS, Regional: combinado raqui-peridural, raquianestesia contínua e raquianestesia

\section{SUMMARY}

Imbelloni LE, Beato L - Comparison between Spinal, Combined Spinal-Epidural and Continuous Spinal Anesthesias for Hip Surgeries in Elderly Patients. A Retrospective Study

Background and Objectives - There are still many questions involving study designs, data analyses and samples size which regard to the demonstration of the benefits of regional anesthesia on patients outcome. Database analysis and data acquisition in general cost less and require less time as compared to large randomized controlled trials. This retrospective study compares continuous spinal anesthesia, combined spinal-epidural and single shot spinal anesthesia for hip surgery in elderly patients during a 4-year period, to determine possible advantages and disadvantages of the three techniques.

Methods - Anesthetic records of 100 patients receiving spinal anesthesia (Group 1), 100 patients receiving combined spinal-epidural block (Group 2) and 100 patients receiving continuous spinal anesthesia (Group 3) over a 4-year period were analyzed. All blockades were performed with patients in the left lateral position. Evaluated parameters were: puncture success, highest level of anesthesia, lower limb motor block, quality of anesthesia, need for additional doses, failures incidence, paresthesia, post-dural puncture headache, cardiovascular changes, mental confusion and delirium, blood transfusion and mortality.

Results - There were no significant differences among groups regarding gender, age, weight and height. Group 2 patients were shorter as compared to groups 1 and 3 . Mean bupivacaine doses were: $15.30 \mathrm{mg}$ in group 1,23.68 mg in group 2 and 10.1 $\mathrm{mg}$ in group 3. They showed statistical significant differences between groups $(p<0.01)$. There were significant differences $(p<0.01)$ in cephalad dispersion between groups 1 and 2, 1 and 3 and 2 and 3 , being lower with continuous spinal and higher with combined spinal-epidural anesthesia. All patients presented total motor block (Bromage score = 3). There were no significant differences in arterial hypotension, bradycardia, paresthesia and blood transfusion. Postoperative mental con- 
fusion was observed in 19 patients, with no difference between techniques. There was no difference in mortality in the first postoperative week and in the first postoperative month.

Conclusions - Retrospective studies usually less and demand less time as compared to controlled studies. This retrospective study has shown that regional anesthesia techniques are related to a low mortality rate in the first postoperative month and to a low incidence of complications.

KEY WORDS - ANESTHETIC TECHNIQUES, Regional: combined epidural spinal block, continuous spinal anesthesia and spinal anesthesia

\section{INTRODUÇÃO}

$\mathrm{D}$ ois procedimentos no quadril, osteossíntese de fratura de fêmur e artroplastia de quadril são freqüentemente realizados em pacientes idosos. Embora existam dados convincentes sobre alguns sistemas específicos (pulmão, coagulação e gastrointestinal), nenhuma técnica em particular tem se mostrado mais segura em pacientes geriátricos submetidos à cirurgia ortopédica de grande porte. Quando comparada com a anestesia geral, a anestesia regional reduz a incidência de mortalidade em aproximadamente $30 \%{ }^{1}$. A utilização de anestesia regional foi associada com uma baixa incidência de mortalidade dentro de 30 dias em diversos estudos aleatórios ${ }^{1}$. Além disso, a anestesia regional diminuiu a trombose venosa profunda em $44 \%$, o embolismo pulmonar em $55 \%$, a transfusão sangüínea em $50 \%$, a pneumonia em $39 \%$, a depressão respiratória em $59 \%$, o infarto agudo do miocárdio em $33 \%$ e a insuficiência renal em $43 \%{ }^{1}$. Desta forma, parece que a utilização de anestesia regional diminui globalmente as complicações pós-operatórias em estudos com grande população de pacientes cirúrgicos.

A injeção de anestésicos locais no espaço subaracnóideo foi realizada pela primeira vez em $1898^{2}$, e em dose única proporciona um bloqueio de instalação rápida e potente. Entretanto, neste bloqueio é difícil a previsão de sua extensão e duração, já que diversos fatores regulam a cinética dos anestésicos locais no líquido cefalorraquidiano (LCR). Araquianestesia contínua foi introduzida em $1907^{3}$, desenvolvida na década de $40^{4,5}$ e investigada clinicamente nas décadas de 60 e $70^{6-9}$. A raquianestesia contínua consiste em introduzir um cateter no espaço subaracnóideo e manter o bloqueio por repetidas injeções de anestésicos. A possibilidade de fracionar a quantidade de anestésico local injetada no tempo permite uma anestesia de instalação rápida, melhor qualidade de bloqueio com menores alterações hemodinâmicas ${ }^{10-12}$. O bloqueio combinado raqui-peridural é uma técnica na qual a raquianestesia e o cateter peridural são simultaneamente utilizados no mesmo paciente. Este bloqueio tem sido usado em cirurgias ortopédicas de grande porte ${ }^{13-15}$

O objetivo deste estudo retrospectivo é comparar as três técnicas anestésicas em cirurgias ortopédicas de grande porte num período de quatro anos, com o objetivo de determinar as possíveis vantagens e desvantagens das técnicas em pacientes ortopédicos idosos.

Revista Brasileira de Anestesiologia

Vol. 52, № 3, Maio - Junho, 2002

\section{MÉTODO}

Foram selecionados e analisados retrospectivamente 521 prontuários de pacientes submetidos a cirurgias de osteossíntese de fêmur ou prótese parcial e total de quadril sob raquianestesia simples, bloqueio combinado raqui-peridural e raquianestesia contínua de Janeiro de 1997 a Dezembro de 2000.

Os critérios de inclusão (Quadro I) no estudo foram: pacientes com idade acima de 60 anos, não internados em UTI, que não fizeram uso de cateter vesical no pré-operatório, estado físico ASA I ou II, operados nas primeiras 24 horas de internação, com os bloqueios realizados em decúbito lateral esquerdo, sem uso de heparina de baixo peso ou opióides subaracnóideo ou peridural, que receberam analgesia pré ou pós-operatória com bloqueio 3 em 1 e que até o momento da fratura tinham uma vida compatível com a idade e sem confusão mental. Todas as anestesias foram realizadas pelos autores.

\section{Quadro I - Critérios de Inclusão no Estudo}

1. Idade acima de 60 anos

2. Estado físico ASA I e II

3. Operados nas primeiras 24 horas de fratura

4. Bloqueios realizados em decúbito lateral esquerdo

5. Não fossem internados na UTI

6. Não tivessem cateterismo vesical

7. Não recebessem heparina de baixo peso molecular antes da cirurgia

8. Não recebessem opióides por via subaracnóidea ou peridural

9. Analgesia pré-operatória ou pós-operatória com bloqueio 3 em 1

10. Não tivessem relato de confusão metal

11. Até o momento da fratura vida compatível com a idade

Nenhum paciente recebeu medicação pré-anestésica no quarto. A monitorização na sala de operações foi realizada pelo ECG contínuo na posição CM5, pressão arterial por método não invasivo e oximetria de pulso. Em todos os pacientes foi puncionada uma veia de membro superior com cateter venoso $18 \mathrm{G}$ e colocado cateter de oxigênio $2 \mathrm{~L}$. $\mathrm{min}^{-1}$ ou máscara de Hudson. Após punção venosa, midazolam (1,5-4,5 mg), meperidina (10-30 mg) foram administrados por via venosa. Para colocação do paciente na posição de bloqueio (DLE), foi injetado cetamina 10 a $30 \mathrm{mg}$ por via venosa ou realizado bloqueio 3 em 1 com $40 \mathrm{ml}$ de bupivacaína a $0,25 \%$. A analgesia pós-operatória foi realizada com $40 \mathrm{ml}$ de bupivacaína a $0,25 \%$ através de bloqueio 3 em 1 e continuada por injeção pelo cateter colocado no espaço perivascular inguinal.

\section{Grupo 1 - Raquianestesia com Dose Única (Raqui Simples)}

Com o paciente em decúbito lateral esquerdo a punção subaracnóidea foi realizada nos espaços $L_{2}-L_{3}$ ou $L_{3}-L_{4}$, usando agulhas descartáveis 27G tipo Quincke (B. Braun Melsungen $A G)$, por via paramediana e com o bisel paralelo às fibras 
da duramáter. Uma vez obtido o LCR, foram injetados $15 \mathrm{mg}$ de bupivacaína a $0,5 \%$ isobárica sem barbotagem, na velocidade de $1 \mathrm{ml} .15 \mathrm{~s}^{-1}$, sendo os pacientes colocados imediatamente em decúbito dorsal (Tabela I). No caso de falha de bloqueio, confirmada por ausência de sensibilidade perineal, foi realizada nova punção e injetada dose menor do mesmo anestésico.

\section{Grupo 2 - Bloqueio Combinado Raqui-Peridural (BCRP)}

Com o paciente em decúbito lateral esquerdo, a punção peridural foi realizada pela via paramediana nos espaços $L_{2}-L_{3}$ ou $L_{3}-L_{4}$, identificado pela perda da resistência à injeção de ar, com conjunto próprio de punção única (Espocan ${ }^{\circledR} \mathrm{B}$. Braun Melsungen $A G)$. Em seguida a agulha de raquianestesia $27 \mathrm{G}$ foi introduzida através da agulha de Tuohy e, após o enchimento do canhão da agulha com LCR, injetava-se bupivacaína a $0,5 \%$ isobárica em dose que dependeu da altura do paciente (Tabela I). Aagulha de raquianestesia era retirada e introduzia-se o cateter peridural. Em caso de dor ou nível insuficiente, $5 \mathrm{ml}$ de bupivacaína a $0,5 \%$ eram injetados pelo cateter peridural. No final da cirurgia o cateter era retirado.

\section{Grupo 3 - Raquianestesia Contínua (Raqui-Contínua)}

Usando técnica descrita anteriormente ${ }^{11}$, a punção peridural foi realizada em decúbito lateral esquerdo, pela via paramediana, nos espaços $L_{2}-L_{3}$ ou $L_{3}-L_{4}$ com agulha $18 \mathrm{G}$ Crawford. Em seguida a duramáter foi puncionada com o conjunto Spinocath $^{\circledR}$ (B.Braun Melsungen AG) com agulha $27 \mathrm{G}$ e cateter 22G. Com o paciente ainda na posição de punção, foi administrada uma dose de 5 a $10 \mathrm{mg}$ de bupivacaína 0,5\% isobárica, dependendo da altura do paciente, que, em seguida, foi colocado na posição supina. Em caso de dor ou nível insuficiente, $2,5 \mathrm{mg}$ de bupivacaína a 0,5\% eram injetados pelo cateter subaracnóideo (Tabela I). No final da cirurgia o cateter era retirado.

O nível de bloqueio sensitivo e o bloqueio motor (escala modificada de Bromage, $0=$ sem bloqueio e $3=$ bloqueio completo) foram testados com intervalos de 5 até 20 minutos. Foram anotadas as doenças associadas assim como as drogas utilizadas.

Os seguintes dados foram analisados: idade, sexo, peso, altura e estado físico de acordo com a classificação da ASA. Fatores da anestesia foram relacionados, como falha do bloqueio, necessidade de complementação com anestesia geral, incidência de hipotensão arterial e bradicardia, necessidade de vasopressor, necessidade de cateterismo vesical, confusão mental, reposição sangüínea e mortalidade em uma semana e um mês.

A sedação foi obtida com doses fracionadas de midazolam e meperidina.

Todos os valores estão expressos em média e DP. Foram utilizados os seguintes testes Kruskal-Wallis, análise de variância, teste de Bonferroni e não paramétrico de Qui-quadrado. Ovalor de $p<0,05$ foi considerado como significativo.

\section{RESULTADOS}

Trezentos pacientes foram selecionados por cumprirem os critérios de inclusão no estudo, sendo 100 de cada técnica apresentada na tabela I. Adose média foi de $15,30 \mathrm{mg}$ de bupivacaína no grupo Raqui Simples, 23,68 mg no grupo BCRP e 10,10 mg na Raqui Contínua, com diferença significativa $(p<0,01)$ entre os grupos 1 e 2,1 e 3 e 2 e 3 , sendo menor com a Raqui Contínua e maior com o BCRP.

As características dos pacientes estão na tabela II, não existindo diferença significativa entre os três grupos em relação a idade, peso e sexo. Os pacientes do grupo 2 foram estatisticamente mais baixos do que os do grupo 1 e 3 . Condições clínicas dos pacientes e drogas utilizadas estão na tabela III, não existindo diferença significativa entre os três grupos.

Tabela I - Doses de Bupivacaína a 0,5\% Preconizadas e Utilizadas

\begin{tabular}{lccc}
\hline & $\begin{array}{c}\text { Raqui } \\
\text { Simples } \\
(\mathrm{n}=100)\end{array}$ & $\begin{array}{c}\text { BCRP } \\
(\mathrm{n}=100)\end{array}$ & $\begin{array}{c}\text { Raqui } \\
\text { Continua } \\
(\mathrm{n}=100)\end{array}$ \\
\hline Altura $<150 \mathrm{~cm}$ & $15 \mathrm{mg}$ & $10 \mathrm{mg}$ & $5 \mathrm{mg}$ \\
Altura entre $151 \mathrm{e} 160 \mathrm{~cm}$ & $15 \mathrm{mg}$ & $12,5 \mathrm{mg}$ & $7,5 \mathrm{mg}$ \\
Altura $>160 \mathrm{~cm}$ & $15 \mathrm{mg}$ & $15 \mathrm{mg}$ & $10 \mathrm{mg}$ \\
Doses adicionais & $10 \mathrm{mg}$ & $25 \mathrm{mg}$ & $2,5 \mathrm{mg}$
\end{tabular}
(nova punção)

Doses utilizadas $(\mathrm{mg})$ * $\quad 15,30 \pm 1,71 \quad 23,68 \pm 23,96 \quad 10,10 \pm 3,19$ **

* Média $\pm \mathrm{DP} ;{ }^{* *} \mathrm{p}<0,01$

Tabela II - Dados Demográficos

\begin{tabular}{lccc}
\hline & $\begin{array}{c}\text { Raqui } \\
\text { Simples } \\
(\mathrm{n}=100)\end{array}$ & $\begin{array}{c}\text { BCRP } \\
(\mathrm{n}=100)\end{array}$ & $\begin{array}{c}\text { Raqui } \\
\text { Continua } \\
(\mathrm{n}=100)\end{array}$ \\
\hline Idade (anos) * & $76,47 \pm 9,38$ & $75,97 \pm 7,58$ & $76,28 \pm 9,72$ \\
Peso $(\mathrm{kg}){ }^{*}$ & $68,63 \pm 13,59$ & $66,85 \pm 11,74$ & $67,85 \pm 12,90$ \\
Altura $(\mathrm{cm}){ }^{*}$ & $164,45 \pm 9,88$ & $161,31 \pm 7,52$ & $164,68 \pm 9,28$ \\
Sexo & & & \\
$\quad$ Masculino & 42 & 34 & 37 \\
Feminino & 58 & 66 & 63 \\
\hline
\end{tabular}

* Valores expressos pela Média \pm DP

Ocorreram três falhas no grupo Raqui Simples, sendo realizada nova punção. No grupo BCRP também ocorreram três falhas, sendo a cirurgia realizada com anestésico injetado pelo cateter peridural. No grupo Raqui Continua não se observou falha de bloqueio. Em 20 pacientes no grupo BCRP e em 15 pacientes no grupo Raqui Contínua foi necessária complementação pelo cateter para início ou término da cirurgia. Todos os pacientes foram sedados com doses fracionadas de midazolam e meperidina, não sendo necessária complementação com anestesia geral.

A dispersão cefálica da analgesia está na tabela IV e mostra diferença significativa $(p<0,01)$ entre os grupos 1 e 2,1 e 3 e 2 e 3. No grupo Raqui Simples, $75 \%$ dos pacientes apresentaram dispersão entre $\mathrm{T}_{10}$ e $\mathrm{T}_{8}$; no grupo $\mathrm{BCRP}, 77 \%$ dos pacientes entre $\mathrm{T}_{10}$ e $\mathrm{T}_{7}$, e na Raqui Contínua $90 \%$ dos pacientes entre $T_{12}$ e $T_{10}$, mostrando uma dispersão mais uniforme com a raquianestesia contínua.

Revista Brasileira de Anestesiologia Vol. 52, No 3, Maio - Junho, 2002 
Tabela III - Condições Clínicas dos Pacientes e Drogas Utilizadas

\begin{tabular}{cccc}
\hline Radas & Raqui & BCRP & Raqui \\
Simples & & Continua \\
& $(\mathrm{n}=100)$ & $(\mathrm{n}=100)$ & $(\mathrm{n}=100)$ \\
\hline
\end{tabular}

\begin{tabular}{llll}
\hline Doenças associadas & & & \\
Hipertensão arterial & 35 & 09 & 38 \\
Distúrbios de ritmo & 08 & 09 & 06 \\
Diabete & 07 & 06 & 06 \\
Doença de Parkinson & 08 & 13 & 14 \\
Testemunhas de Jeová & 16 & & \\
\hline Drogas utilizadas & & 49 & 48 \\
Antihipertensivos & 52 & 28 & 25 \\
Diuréticos & 26 & 10 & 11 \\
Antidisrítmicos & 08 & 06 & 0 \\
Broncodilatadores & 05 & & \\
\hline
\end{tabular}

Tabela IV - Dispersão Cefálica da Analgesia

\begin{tabular}{lccc}
\hline & $\begin{array}{c}\text { Raqui } \\
\text { Simples } \\
(\mathrm{n}=100)\end{array}$ & $\begin{array}{c}\text { BCRP } \\
(\mathrm{n}=100)\end{array}$ & $\begin{array}{c}\text { Raqui } \\
\text { Continua } \\
(\mathrm{n}=100)\end{array}$ \\
\hline $\mathrm{T}_{5}$ & 0 & 3 & 0 \\
$\mathrm{~T}_{6}$ & 1 & 8 & 0 \\
$\mathrm{~T}_{7}$ & 8 & 21 & 1 \\
$\mathrm{~T}_{8}$ & 29 & 26 & 3 \\
$\mathrm{~T}_{9}$ & 14 & 19 & 6 \\
$\mathrm{~T}_{10}$ & 32 & 11 & 28 \\
$\mathrm{~T}_{11}$ & 6 & 6 & 29 \\
$\mathrm{~T}_{12}$ & 10 & 6 & 33 \\
Moda & $\mathrm{T}_{10}$ & $\mathrm{~T}_{8}$ & $\mathrm{~T}_{12}$ \\
\hline
\end{tabular}

Todos os pacientes apresentaram bloqueio motor completo. Não houve diferença significativa em relação a hipotensão arterial, bradicardia, parestesia e necessidade de sangue. Dezenove pacientes apresentaram confusão mental no pós-operatório sem diferença entre as técnicas utilizadas (Tabela V).

Não há diferença significativa na incidência de óbitos na primeira semana e no primeiro mês de pós-operatório.

\section{DISCUSSÃO}

O número de pacientes não estudados ou excluídos do estudo é grande. Fichas incompletas podem resultar em limitações dos estudos retrospectivos ${ }^{16}$. Este estudo mostrou que para cirurgias ortopédicas de grande porte no paciente idoso, a raquianestesia contínua apresenta algumas vantagens sobre a raquianestesia simples ou o bloqueio combinado raqui-peridural: menor dispersão cefálica, menor incidência de hipotensão arterial e menor dose de anestésico local.

Revista Brasileira de Anestesiologia

Vol. 52, Nº 3, Maio - Junho, 2002
Tabela V - Complicações

\begin{tabular}{lccc}
\hline & $\begin{array}{c}\text { Raqui } \\
\text { Simples } \\
(\mathrm{n}=100)\end{array}$ & $\begin{array}{c}\text { BCRP } \\
(\mathrm{n}=100)\end{array}$ & $\begin{array}{c}\text { Raqui } \\
\text { Continua } \\
(\mathrm{n}=100)\end{array}$ \\
\hline Hipotensão arterial & 10 & 14 & 5 \\
Bradicardia & 4 & 5 & 5 \\
Parestesia & 2 & 2 & 4 \\
Transfusão & 6 & 6 & 5 \\
Sondagem vesical & 4 & 5 & 2 \\
Confusão mental & 7 & 8 & 4 \\
Óbito 1 semana & 2 & 4 & 1 \\
Óbito 1 mês & 3 & 6 & 2 \\
Complicações neurológicas & 0 & 0 & 0 \\
Cefaléia & 0 & 0 & 0 \\
\hline
\end{tabular}

Fraturas de fêmur e de quadril têm grande importância nos serviços de saúde. A incidência aumenta com a idade, refletindo um predomínio das mulheres em razão das fraturas associadas com osteoporose. No presente estudo este fato foi confirmado pela incidência de $62 \%$ de mulheres.

Os pacientes de bloqueio combinado raqui-peridural e de raquianestesia contínua receberam menor dose inicial de bupivacaína isobárica do que os pacientes de raquianestesia em dose única, em virtude da presença do cateter que facilitaria o aumento da dose quando necessário. Isto teve como conseqüência uma complementação pelo cateter em $20 \%$ no bloqueio combinado raqui-peridural e $15 \%$ na raquianestesia contínua sem necessidade de complementação com anestesia geral.

O nível sensitivo pode ser facilmente obtido e controlado com a raquianestesia contínua, enquanto que uma dose maioré necessária quando se utiliza a raquianestesia em dose única, da mesma forma que quando se utiliza a anestesia peridural. Aestabilidade cárdio-circulatória observada nos três grupos com a solução isobárica, mostra que pode haver uma dissociação entre o nível de bloqueio sensitivo e o bloqueio simpático. O nível da analgesia com a raquianestesia contínua ficou dois segmentos abaixo da raquianestesia em dose única e quatro segmentos abaixo do bloqueio combinado raqui-peridural.

A escala de Bromage é um indicador qualitativo do bloqueio motor e não o quantifica. Entretanto, como todos os pacientes apresentaram o grau máximo de bloqueio motor dos membros inferiores após a injeção do anestésico, não foi necessário correlacionar seus diferentes graus. Uma interessante vantagem das três técnicas para cirurgias ortopédicas de grande porte é a utilização inicial do anestésico no espaço subaracnóideo, resultando num bloqueio motor completo com baixas doses, o que dificilmente se obteria com a anestesia peridural pura.

Uma das maiores razões para a preferência do bloqueio peridural é a hipotensão arterial precoce e rápida causada pela raquianestesia. Aestabilidade da anestesia periduralé puramente histórica ${ }^{17}$. Neste estudo, o anestésico local foi injetado no espaço subaracnóideo em todos os pacientes, mas no 
grupo do bloqueio combinado raqui-peridural a necessidade de complementação resultou numa maior incidência de hipotensão arterial, em comparação com os dois outros grupos, porém sem significância estatística. A estabilidade da pressão arterial é um problema nos pacientes idosos, independente da técnica utilizada. Entretanto, em 300 pacientes a incidência de hipotensão arterial foi menor do que $10 \%$. Um importante achado deste estudo foi a baixa incidência de retenção urinária que necessitasse de cateterização vesical $(3,6 \%)$. A retenção urinária deve-se a mecanismos neurogênicos periféricos e centrais controlando o reflexo da micção, incluindo o bloqueio autonômico, cujo tempo para desaparecimento é três vezes maior do que o tempo de regressão de dois segmentos ${ }^{18}$. Neste estudo, a cateterização da bexiga ocorreu quando os bloqueios já tinham desaparecido em todos os pacientes. Apenas 11 pacientes necessitaram de cateterismo vesical, e isto é importante no contexto das próteses parciais e totais de quadril. Sepsis após artroplastia usualmente resulta em rejeição da prótese. Bacteremia pode seguir à instrumentação uretral e a necessidade de cateterização aumenta em $6,2 \%$ a incidência de sepsis pós- artroplastia ${ }^{19}$. Por proporcionar um nível de bloqueio mais baixo e menor dose, a raquianestesia contínua cursou com menor incidência de necessidade de cateterismo vesical.

Confusão mental no pós-operatório é uma situação transitória no idoso e sua etiologia é desconhecida. Diversos estudos de fratura de quadril não conseguiram correlacionar a confusão mental com o tipo de anestesia utilizada. Existem vários fatores independentes para o aparecimento de delírio pós-operatório, incluindo dor pós-operatória ${ }^{16}$. Em razão da anestesia regional proporcionar uma analgesia superior, quando comparada com técnicas sistêmicas, ela pode ter algumas vantagens na diminuição de confusão mental no pós-operatório. Aanalgesia deste grupo de pacientes foi realizada através do bloqueio 3 em 1 , administrado antes ou após o bloqueio subaracnóideo ${ }^{20}$. A incidência de confusão mental foi de $6,3 \%$ e desapareceu no segundo ou terceiro dia de pós-operatório. Apesar de não haver diferença significativa, a confusão mental apareceu a metade das vezes com a raquianestesia contínua em comparação com a raquianestesia simples e o bloqueio combinado raqui-peridural.

Quando comparada com a anestesia geral, a anestesia regional reduz a mortalidade em aproximadamente $30 \%{ }^{1}$. A anestesia regional está associada a uma baixa incidência de mortalidade dentro dos primeiros 30 dias, em que $75 \%$ das mortes são devidas a embolismo pulmonar, infecção e problemas cardíacos ${ }^{1}$. Recente estudo com 450 pacientes mostrou uma incidência de morte de $3 \%$ até o $14^{\circ}$ dia de pós-operatório após raquianestesia, em comparação com $7 \%$ após anestesia geral ${ }^{21}$. No presente estudo não se comparou a incidência de morte com a anestesia geral, mas mostrou que a anestesia regional cursou com praticamente a mesma incidência de morte (3,6\%). O aumento da morbi-mortalidade em idosos é explicado pelo declínio na função orgânica básica do paciente. Ao estudar a incidência de óbito nas primeiras 24 horas de pós-operatório em pacientes acima de 65 anos, foi encontrada uma baixa incidência $(1,38 \%)$, sem no entanto se correlacionar com o tipo de cirurgia ${ }^{22}$. Da mesma forma que a necessidade de sondagem vesical e da hipotensão arterial, a incidência de mortalidade na primeira semana e no primeiro mês foi a metade com a raquianestesia contínua quando comparada à raquianestesia simples e ao bloqueio combinado raqui-peridural.

Diversos trabalhos têm demonstrado os benefícios da anestesia regional quando ela é estendida ao pós-operatório ${ }^{20,23}$. Entretanto, a analgesia pós-operatória é apenas um dos fatores avaliados nos estudos de morbi-mortalidade. Estudo realizado com bloqueio do plexo lombar ( 3 em 1 ) no pós-operatório ${ }^{20}$ mostrou uma analgesia de melhor qualidade, com um tempo médio em torno de 16 horas ${ }^{20}$ e menor necessidade de opióides ${ }^{20}$ com baixa utilização de cateter vesical, podendo ter contribuído para uma baixa mortalidade em um mês $(3,6 \%)$.

Os estudos prospectivos controlados apresentam resultados mais fidedignos, mas para determinar se a anestesia regional tem algum benefício na mortalidade seria necessária uma população de 24.000 pacientes ${ }^{24}$. Apesar do aumento do uso de meta-análises, que combinam e avaliam dados de diferentes procedências, elas apresentam algumas desvantagens ${ }^{25}$. Estudos retrospectivos geralmente custam pouco e requerem menos tempo quando comparados com estudos controlados, podendo apresentar resultados não fidedignos ${ }^{26}$. O presente estudo é uma avaliação retrospectiva, mas um dos primeiros trabalhos no Brasil a correlacionar as técnicas de anestesia regional com a morbi-mortalidade em pacientes idosos com fraturas de quadril. O resultado mostrou uma baixa incidência de mortalidade $(3,6 \%)$ no primeiro mês e baixa incidência de complicações com o uso das três técnicas de anestesia regional.

\section{Comparison between Spinal, Combined Spinal-Epidural and Continuous Spinal Anesthesias for Hip Surgeries in Elderly Patients. A Retrospective Study}

\author{
Luiz Eduardo Imbelloni, TSA, M.D., Lúcia Beato, M.D.
}

\section{INTRODUCTION}

Femur fracture osteosynthesis and hip artroplasty are common procedures in elderly patients. Despite some consistent data regarding specific systems (lung, coagulation and GI tract), no single anesthetic technique has proved to be safer in geriatric patients submitted to major orthopedic surgeries. When compared to general anesthesia, regional anesthesia decreases mortality in approximately $30 \%{ }^{1}$. Several randomized studies have associated regional anesthesia to a low mortality rate in 30 days ${ }^{1}$. In addition, regional anesthesia decreased deep vein thrombosis in $44 \%$, pulmonary embolism in $55 \%$, blood transfusion in $50 \%$, pneumonia in $39 \%$, 
respiratory depression in 59\%, acute myocardial infarction in $33 \%$ and renal failure in $43 \%{ }^{1}$. So it seems that regional anesthesia has globally reduced postoperative complications in studies with large surgical patients populations.

Subarachnoid injection of local anesthetic was first performed in $1898^{2}$. In a single dose, it provides a potent blockade of fast onset, however with extension and duration difficult to estimate since several factors regulate local anesthetics kinetics in the CSF. Continuous spinal anesthesia was introduced in $1907^{3}$, developed during the $40 \mathrm{~s}^{4,5}$ and clinically investigated in the $60 \mathrm{~s}$ and $70 \mathrm{~s}^{6-9}$. Continuous spinal anesthesia consists of introducing a catheter in the subarachnoid space and maintaining blockade by repeated anesthetic injections. The possibility of fractionating local anesthetics dosage along time allows a fast onset with better blockade quality and less hemodynamic changes ${ }^{10-12}$. Combined spinal-epidural anesthesia is a technique in which spinal anesthesia and epidural catheter are simultaneously used. This blockade has been used in major orthopedic surgeries ${ }^{13-15}$. This retrospective study aimed at comparing these three anesthetic techniques in major orthopedic surgeries throughout a 4-year period to determine their possible advantages and disadvantages in elderly patients.

\section{METHODS}

Anesthetic records of 521 patients submitted to femur osteosynthesis and total or partial hip replacement under single shot spinal anesthesia, combined spinal-epidural block and continuous spinal anesthesia between January 1997 and December 2000 were selected and retrospectively analyzed.

Inclusion criteria (Chart I) were: patients aged above 60 years, not admitted to ICU, without preoperative vesical catheter, physical status ASA I or II, submitted to surgery within the first 24 hours after admission, with blockades performed in the left lateral position, not receiving low molecular weight heparin, spinal or epidural opioids, who received pre or postoperative analgesia with the 3 in 1 blockade and who, until the fracture, had a life compatible with age and without mental confusion. All anesthesias were performed by the authors.

\section{Chart I - Inclusion Criteria}

1. More than 60 years of age

2. Physical status ASA I and II

3. Submitted to surgery within 24 hours after fracture

4. Blockades performed in the left lateral position

5. Not admitted to ICU

6. Absence of vesical catheter

7. Not receiving low molecular weight heparin before surgery

8. Not receiving spinal or epidural opioids

9. Pre or postoperative analgesia with 3 in 1 blockade

10. No history of mental confusion

11. Life compatible with age until fracture
No patient was premedicated. Monitoring in the operating room consisted of continuous ECG in CM5, non-invasive blood pressure and pulse oximetry. All patients had an upper limb vein punctured with an $18 \mathrm{G}$ venous catheter and a 2 L. min $^{-1}$ oxygen catheter or Hudson mask installed. After venous puncture, patients were given intravenous midazolam (1.5 - $4.5 \mathrm{mg})$ and meperidine (10 - $30 \mathrm{mg})$. To place the patient in the blockade position (LLP), 10 to $30 \mathrm{mg}$ intravenous ketamine were injected, or 3 in 1 blockade was performed with $40 \mathrm{ml}$ of $0.25 \%$ bupivacaine. Postoperative analgesia was achieved with $40 \mathrm{ml}$ of $0.25 \%$ bupivacaine through the 3 in 1 blockade as well, and was maintained by injections through a catheter placed in the inguinal perivascular space.

\section{Group 1 - Single Shot Spinal Anesthesia (Simple Spinal)}

Spinal puncture was paramedially performed at $L_{2}-L_{3}$ or $L_{3}-L_{4}$ interspace with the patient in the left lateral position, using $27 \mathrm{G}$ disposable Quincke needles (B. Braun, Meisungen, $A G)$, with their bevels always parallel to dural fibers. After obtaining CSF, $15 \mathrm{mg}$ of $0.5 \%$ isobaric bupivacaine were injected without barbotage, at a rate of $1 \mathrm{ml} .15 \mathrm{~s}^{-1}$. Patients were then immediately placed in the supine position (Table I). In case of failure, confirmed by lack of perineal sensitivity, a new puncture was performed and a lower dose of the same anesthetic drug was injected.

\section{Group 2 - Combined Spinal-Epidural Block (CSEB)}

With patients in the left lateral position, epidural puncture was paramedially performed at $L_{2}-L_{3}$ or $L_{3}-L_{4}$ interspace using the loss of resistance to air technique with an adequate single shot device (Espocan ${ }^{\circledR}$ B. Braun Meisungen AG). Then, a $27 \mathrm{G}$ spinal anesthesia needle was introduced through the Tuohy needle and, after CSF return, $0.5 \%$ isobaric bupivacaine was injected in a dose depending on patients height (Table I). Spinal needle was then removed and an epidural catheter was inserted. In the presence of pain or inadequate block level, $5 \mathrm{ml}$ of $0.5 \%$ bupivacaine were injected through the epidural catheter, which was removed at the end of the surgery.

\section{Group 3 - Continuous Spinal Anesthesia (Continuous Spinal)}

Using the previously described technique ${ }^{11}$, the epidural puncture was paramedially performed in the left lateral position at $L_{2}-L_{3}$ or $L_{3}-L_{4}$ interspace with an $18 G$ Crawford needle. After that, dura was punctured with a Spinocath ${ }^{\circledR}$ device (B. Braun Meisungen AG) with a $27 \mathrm{G}$ needle and $22 \mathrm{G}$ catheter set. With the patient still in the puncture position, 5 to $10 \mathrm{mg}$ of $0.5 \%$ isobaric bupivacaine were injected, depending on patients' height, when they were immediately placed in the supine position. In case of pain or inadequate level, $2.5 \mathrm{mg}$ of $0.5 \%$ bupivacaine were injected through the spinal catheter (Table I), which was removed at the end of surgery.

Sensory block level and motor block degree (modified Bromage scale, $0=$ no blockade and $3=$ total blockade) were tes- 
ted at 5 to 20-minute intervals. Associated diseases and drugs in use were also recorded.

The following data were analyzed: age, gender, height, weight and ASA physical status. Anesthesia mishappenings, such as blockade failure, need to complement with general anesthesia, arterial hypotension and bradycardia, need for vasopressants or vesical catheter, mental confusion, blood transfusion and 1 week/ 1 month mortality were recorded. Sedation was obtained with fractionated midazolam and meperidine doses.

All values are expressed in mean and SD. The following statistical tests were used: Kruskal-Wallis, analysis of variance, Bonferroni's test and non-parametric Chi-square test, considering significant $p<0.05$.

\section{RESULTS}

Three hundred patients' records which complied with the inclusion criteria were studied, with 100 cases following each technique presented in table I. Mean bupivacaine dose was $15.30 \mathrm{mg}$ in the Simple Spinal Group, $23.68 \mathrm{mg}$ in the CSEB Group and $10.10 \mathrm{mg}$ in the Continuous Spinal Group, with statistical significant differences $(p<0.01)$ between groups 1 and 2,1 and 3 , and 2 and 3 .

Patients demographics data are shown in table II, with no statistical differences between groups in age, weight and gender. Group 2 patients were statistically shorter as compared to groups 1 and 3 . Patients clinical conditions and drugs in use are shown in table III, with no significant differences between groups.

Table I - Recommended and Used 0.5\% Bupivacaine Doses

\begin{tabular}{lccc}
\hline & $\begin{array}{c}\text { Single Shot } \\
\text { Spinal } \\
(\mathrm{n}=100)\end{array}$ & $\begin{array}{c}\text { CSEB } \\
(\mathrm{n}=100)\end{array}$ & $\begin{array}{c}\text { Continuous } \\
\text { Spinal } \\
(\mathrm{n}=100)\end{array}$ \\
\hline Height $<150 \mathrm{~cm}$ & $15 \mathrm{mg}$ & $10 \mathrm{mg}$ & $5 \mathrm{mg}$ \\
$\begin{array}{l}\text { Height between } 151 \\
\text { and } 160 \mathrm{~cm}\end{array}$ & $15 \mathrm{mg}$ & $12.5 \mathrm{mg}$ & $7.5 \mathrm{mg}$ \\
Height $>160 \mathrm{~cm}$ & $15 \mathrm{mg}$ & $15 \mathrm{mg}$ & $10 \mathrm{mg}$ \\
Additional doses & $10 \mathrm{mg}$ & $25 \mathrm{mg}$ & $2.5 \mathrm{mg}$
\end{tabular}

(new puncture)

Doses used $(\mathrm{mg})$ *

$15.30 \pm 1.7123 .68 \pm 23.96 \quad 10.10 \pm 3.19$ **

* Values expressed in Mean \pm SD; ** $p<0.01$

Table II - Demographics Data

\begin{tabular}{lccc}
\hline & $\begin{array}{c}\text { Single Shot } \\
\text { Spinal } \\
(\mathrm{n}=100)\end{array}$ & $\begin{array}{c}\text { CSEB } \\
(\mathrm{n}=100)\end{array}$ & $\begin{array}{c}\text { Continuous } \\
\text { Spinal } \\
(\mathrm{n}=100)\end{array}$ \\
\hline Age (years) ${ }^{*}$ & $76.47 \pm 9.38$ & $75.97 \pm 7.58$ & $76.28 \pm 9.72$ \\
Weight $(\mathrm{kg}){ }^{*}$ & $68.63 \pm 13.59$ & $66.85 \pm 11.74$ & $67.85 \pm 12.90$ \\
Height $(\mathrm{cm}){ }^{*}$ & $164.45 \pm 9.88$ & $161.31 \pm 7.52$ & $164.68 \pm 9.28$ \\
Gender & & & \\
Male & 42 & 34 & 37 \\
Female & 58 & 66 & 63 \\
\hline
\end{tabular}

*Values expressed in Mean \pm SD
Table III - Patients' Clinical Conditions and Drugs in Use

\begin{tabular}{lccc}
\hline & $\begin{array}{c}\text { Single Shot } \\
\text { Spinal } \\
(\mathrm{n}=100)\end{array}$ & $\begin{array}{c}\text { CSEB } \\
(\mathrm{n}=100)\end{array}$ & $\begin{array}{c}\text { Continuous } \\
\text { Spinal } \\
(\mathrm{n}=100)\end{array}$ \\
\hline Associated diseases & & & \\
$\quad$ Arterial hypertension & 35 & 33 & 38 \\
Rhythm abnormalities & 08 & 09 & 06 \\
Diabetes & 07 & 09 & 09 \\
Parkinson's disease & 08 & 06 & 06 \\
Jehovah Witnesses & 16 & 13 & 14 \\
\hline Drugs used & & & \\
Antihipertensive & 52 & 49 & 48 \\
Diuretics & 26 & 28 & 25 \\
Antiarhythmic & 08 & 10 & 11 \\
Broncodilators & 05 & 06 & 0 \\
\hline
\end{tabular}

There were 3 failures in the Simple Spinal Group with the need for a new puncture. There were also 3 failures in the CSEB group and surgery was performed with more anesthetic being injected through the epidural catheter. There was no failure in the Continuous Spinal group. In 20 CSEB and 15 Continuous Spinal patients, an additional dose via catheter was needed to begin or complete the surgery. All patients were sedated with fractionated midazolam and meperidine doses, with no need for general anesthesia complementation.

Cephalad analgesic spread is shown in table IV, with significant differences $(p<0.01)$ between groups 1 and 2,1 and 3 , and 2 and 3 . In the Simple Spinal group, $75 \%$ of patients showed a spread between $\mathrm{T}_{10}$ and $\mathrm{T}_{8}$; in the CSEB group, $77 \%$ of patients had it between $\mathrm{T}_{10}$ and $\mathrm{T}_{7}$ and in the Continuous Spinal group, $90 \%$ of patients presented a spread between $T_{12}$ and $T_{10}$. This last group showed therefore a more uniform spread of the anesthetic.

Table IV - Analgesia Cephalad Spread

\begin{tabular}{lccc}
\hline & $\begin{array}{c}\text { Single Shot } \\
\text { Spinal } \\
(\mathrm{n}=100)\end{array}$ & $\begin{array}{c}\text { CSEB } \\
(\mathrm{n}=100)\end{array}$ & $\begin{array}{c}\text { Continuous } \\
\text { Spinal } \\
(\mathrm{n}=100)\end{array}$ \\
\hline $\mathrm{T}_{5}$ & 0 & 3 & 0 \\
$\mathrm{~T}_{6}$ & 1 & 8 & 0 \\
$\mathrm{~T}_{7}$ & 8 & 21 & 1 \\
$\mathrm{~T}_{8}$ & 29 & 26 & 3 \\
$\mathrm{~T}_{9}$ & 14 & 19 & 6 \\
$\mathrm{~T}_{10}$ & 32 & 11 & 28 \\
$\mathrm{~T}_{11}$ & 6 & 6 & 29 \\
$\mathrm{~T}_{12}$ & 10 & 6 & 33 \\
Mode & $\mathrm{T}_{10}$ & $\mathrm{~T}_{8}$ & $\mathrm{~T}_{12}$ \\
\hline
\end{tabular}

All patients had total motor block.

There were no significant differences in hypotension, bradycardia, paresthesia and blood transfusion. Nineteen patients presented postoperative mental confusion, with no significant difference between techniques (Table V).

Revista Brasileira de Anestesiologia Vol. 52, No 3, Maio - Junho, 2002 
Table V - Complications

\begin{tabular}{lccc}
\hline & $\begin{array}{c}\text { Single Shot } \\
\text { Spinal } \\
(\mathrm{n}=100)\end{array}$ & $\begin{array}{c}\text { CSEB } \\
(\mathrm{n}=100)\end{array}$ & $\begin{array}{c}\text { Continuous } \\
\text { Spinal } \\
(\mathrm{n}=100)\end{array}$ \\
\hline Arterial hypotension & 10 & 14 & 5 \\
Bradycardia & 4 & 5 & 5 \\
Paresthesia & 2 & 2 & 4 \\
Transfusion & 6 & 6 & 5 \\
Vesical catheter & 4 & 5 & 2 \\
Mental confusion & 7 & 8 & 4 \\
Death within 1 week & 2 & 4 & 1 \\
Death within 1 month & 3 & 6 & 2 \\
Neurological complications & 0 & 0 & 0 \\
Headache & 0 & 0 & 0 \\
\hline
\end{tabular}

There were also no significant differences in mortality in the first week and within 1 month after surgery.

\section{DISCUSSION}

The number of patients not studied or excluded from the study is high. Incomplete records may result in retrospective studies limitations ${ }^{16}$.

This study has shown that for major orthopedic surgeries in elderly patients, continuous spinal anesthesia has some advantages over simple spinal or combined spinal-epidural anesthesia: less cephalad spread, lower incidence of arterial hypotension and less local anesthetic requirement.

Femur and hip fractures are major issues for health services. Incidence increases with age, with predominance of women due to association to osteoporosis. In our study, this was confirmed by the $62 \%$ presence of women in the groups.

Combined spinal-epidural and continuous spinal anesthesia patients received a lower initial isobaric bupivacaine dose as compared to single shot spinal anesthesia patients. Due to the presence of the catheter, which would allow dose increment if needed. The consequence was a $20 \%$ catheter complementation in the combined spinal-epidural block and a $15 \%$ complementation in the continuous spinal block with no need for general anesthesia association.

Sensory block level may be easily obtained and controlled with continuous spinal anesthesia, while a higher dose is needed when single shot spinal anesthesia or epidural anesthesia are used. Cardiocirculatory stability seen in all groups with the isobaric solution shows that there might be a dissociation between sensory and sympathetic blocks. Analgesia level with continuous spinal anesthesia remained two segments below single shot spinal anesthesia and 4 segments below combined spinal-epidural block.

Bromage scale is a motor block qualitative but not quantitative indicator. However, since all patients reached the highest lower limbs motor block degree after anesthetic injection, there was no need to correlate different degrees. An interesting advantage of all techniques for major orthopedic surgeries is the initial anesthetic injection in the subarachnoid space resulting in total motor block with low doses, which would be difficult to obtain with epidural anesthesia alone.

One of the major reasons for the preference for epidural blockade is the early and rapid hypotension caused by spinal anesthesia, but epidural anesthesia stability is purely histori$\mathrm{cal}^{17}$. In our study, all patients received spinal local anesthetics, but the epidural additional dose given to the spinal-epidural group resulted in a higher incidence of hypotension as compared to other groups, with no statistical significance though. Blood pressure stability is a problem in elderly patients, regardless to the technique employed. However, the incidence of hypotension among our 300 patients was less than $10 \%$.

An important finding of this study was the low incidence of urine retention requiring vesical catheter (3.6\%). Urine retention is caused by peripheral and central neurogenic mechanisms interfering with micturition reflexes, including autonomic blockade, which takes three times longer to resolve than two segments regression time ${ }^{18}$. In our study, bladder catheterization was needed when blockades had already disappeared in all patients. Only 11 of them needed a vesical catheter and this is important in the partial or total hip replacement context. Post-arthroplasty infection results in general in prosthesis rejection. Bacteremia may follow uretral instrumentation and the need for catheterization increases post-arthroplasty sepsis in $6.2 \%{ }^{19}$. For providing a lower blockade level with a lower local anesthetic dose, continuous spinal anesthesia group showed the lowest vesical catheter need requirement.

Postoperative mental confusion is a transient situation for the elderly and its etiology is not known. Different hip fracture studies were unable to correlate mental confusion to the anesthetic technique employed. There are several independent factors affecting postoperative delirium, including postoperative pain ${ }^{16}$. Since regional anesthesia provides a better analgesia as compared to systemic techniques, it may be advantageous in decreasing postoperative mental confusion. Our patients received analgesia by the 3 in 1 blockade technique administered before or after spinal block ${ }^{20}$. Mental confusion incidence was $6.3 \%$ and disappeared within 1 or 2 postoperative days. Although not achieving statistical significance, mental confusion was seen half the times with spinal anesthesia as compared to single shot spinal anesthesia and combined spinal-epidural block.

Regional anesthesia decreases mortality in approximately $30 \%$ when compared to general anesthesia 1 . Regional anesthesia is associated to a low mortality rate within 30 days, when $75 \%$ of deaths are caused by pulmonary embolism, infection or heart disturbances ${ }^{1}$. A recent study with 450 patients has shown a mortality rate of $3 \%$ within 14 postoperative days after spinal anesthesia, compared to $7 \%$ after general anesthesia ${ }^{21}$. Our study has not compared mortality with general anesthesia, but has shown that regional anesthesia had virtually the same mortality rate (3.6\%). Increased morbidity-mortality in elderly patients is explained by a decrease in patients' basic organic functions. One study found a low mortality rate $(1.38 \%)$ during the first postoperative days 
in patients above 65 years of age, but didn't correlate it to the type of surgery ${ }^{22}$. Similarly to vesical catheter need and arterial hypotension, mortality rates during the first week and the first month were two times lower with continuous spinal anesthesia as compared to single shot spinal anesthesia and combined spinal-epidural block.

Several papers have shown the benefits of regional anesthesia when it is extended to the postoperative period ${ }^{20,23}$. Postoperative analgesia, however, is just one of the factors taken into account by morbidity-mortality studies. A study performed with postoperative lumbar plexus block (3 in 1) ${ }^{20}$ has shown a better analgesia lasting for a mean time of 16 hours ${ }^{20}$, and lower need for opioids ${ }^{20}$ and vesical catheter, which might have contributed to the low mortality rate in one month (3.6\%).

Prospective controlled studies provide more reliable results, but to establish whether regional anesthesia has some benefit on mortality, it would be necessary a sample size of 24,000 patients ${ }^{24}$. In spite of the increased use of meta-analysis combining and evaluating data from different sources, they have some disadvantages ${ }^{25}$. Retrospective studies in general cost less and take less time as compared to controlled studies, but may result in unreliable findings ${ }^{26}$. Our study is a retrospective evaluation and one of the first papers in Brazil correlating regional anesthesia techniques to morbidity-mortality in elderly patients with hip fractures. Our results have shown a low mortality rate $(3.6 \%)$ in the first month and a low complication rate with the three regional anesthesia techniques.

\section{REFERÊNCIAS - REFERENCES}

01. Schug AS - Is Regional Anesthesia Better than General Anesthesia? em: Syllabus of the $24^{\text {th }}$ Annual Meeting of the American Society of Regional Anesthesia, Philadelphia, 1999;62-64.

02 . Bier A - Versuche über cocainisierung des rückenmarkes. Deutsche Z Chi, 1898;51:361-369.

03. Dean HP - Discussion on the relative value of inhalation and injection methods of inducing anaesthesia. BMJ, 1907;2: 869-877.

04. Lemmon WT - A method for continuous spinal anesthesia. Annals of Surgery, 1940;111:141-144.

05. Tuohy EB - Continuous spinal anesthesia: its usefulness and technique involved. Anesthesiology, 1944;5:142-148.

06. Bizzarri D, Giuffrida JG, Bandoc L et al - Continuous spinal anesthesia using a special needle and catheter. Anesth Analg, 1964;43:393-396.

07. Teixeira JW, Borges CS, Burigo FO - Raquianestesia contínua em pacientes de idade avançada. Rev Bras Anestesiol, 1970;20:518-524.

08. Sylos FE, Abbondanza R, Delboni W et al - Raquianestesia contínua em operações ortopédicas de quadril. Rev Bras Anestesiol, 1977;27:153-160.

09. Elam JO - Catheter subarachnoid block for labor and delivery: a differential segmental technich employing hyperbaric lidocaine. Anesth Analg, 1970;49:1007-1015.

10. De Andrés JA, Febré A, Bellver J et al - Continuous spinal anaesthesia versus single dosing. A comparative study. European $\mathrm{J}$ Anaesthesiol, 1995;12:135-140.
11. Imbelloni LE, Gouveia MA - Um novo cateter para raquianestesia contínua. Rev Bras Anestesiol, 1998;48:527-529.

12. Chaoui K, Pertek JP, Lemarie J et al - Rachianesthésie standard et continue chez le patient âgé. Cah Anesthesiol, 1997;45: 407-413.

13. Coates MB - Combined subarachnoid and epidural techniques. Anaesthesia, 1982;37:89-90.

14. Mumtaz MH, Daz M, Kuz M - Combined subarachnoid and epidural techniques. Anaesthesia, 1982;37:90

15. Imbelloni LE, Carneiro ANG - Bloqueio combinado raquiperidural para cirurgias ortopédicas: agulha de dupla luz em punção única ou duas agulhas em espaços diferentes. Rev Bras Anestesiol, 1998:48:177-183.

16. Wu CL, Fleisher LA - Outcomes research in regional anesthesia and analgesia. Int Anesth Res S, 2000;91:1232-1242.

17. Crawford JS - There is only a limited place for spinals in obstetrics. Acta Anaesthesiol Bel, 1988;39:181-184.

18. Phillipe AC, Durart MD - Intraspinal analgesia and urinary bladder dysfunction. Acta Anaesthesiol Belg, 1988;39:163-167.

19. Wroblweski BM - Urethal instrumentation and deep sepsis in total hip replacement. CORR, 1980;146:209-212.

20. Imbelloni LE, Gouveia MA - Analgesia pós-operatória com bloqueio do plexo lombar: comparação entre as técnicas com cateter e com estimulação do nervo femoral. Rev Bras Anestesiol, 2001:51:28-36.

21. Fahmy $\mathrm{N}$ - Does anesthesia influence the outcome of femoral neck fractures in the elderly? Anesthesiology, 1998;89:A819.

22. Cicarelli DD, Gotardo AOM, Auler Jr JOC et al - Incidência de óbitos anestésicos-cirúrgicos nas primeiras 24 horas. Revisão de prontuários de 1995 no Hospital das Clínicas da FMUSP. Rev Bras Anestesiol, 1998;48:289-294.

23. Liu S, Carpenter RL, Neal JM - Epidural anesthesia and analgesia: their role in postoperative outcome. Anesthesiology, 1995;82:1474-1506.

24. Bode Jr RH, Lewis KP, Zarich SW et al - Cardiac outcome after peripheral vascular surgery: comparison of general and regional anesthesia. Anesthesiology, 1996;84:3-13.

25. LeLorier J, Gregoire G, Benhaddad A et al - Discrepancies between meta-analyses and subsequent large randomized controlled trials. N Engl J Med, 1997;337-536-542.

26. lezzoni LI - Assessing quality using administrative data. Ann Intern Med, 1997;127:666-674.

\section{RESUMEN}

Imbelloni LE, Beato L - Comparación entre Raquianestesia, Bloqueo Combinado Raqui-Peridural y Raquianestesia Continua para Cirugías de Cuadril en Pacientes Ancianos. Estudio Retrospectivo

Justificativa y Objetivos - Diversas cuestiones envuelven los estudios, las análisis y el tamaño de la muestra para que sean demostrados los beneficios de la anestesia regional. Las análisis de datos generalmente cuestan menos y requieren menos tiempo, cuando comparado con un amplio estudio aleatorio controlado. Esta análisis retrospectiva compara la raquianestesia continua, el bloqueo combinado raqui-peridural y la raquianestesia simple para cirugías de cuadril en pacientes ancianos durante cuatro años, para determinar las posibles ventajas y desventajas de las tres técnicas.

Método - Fueron evaluadas 300 fichas siendo que: 100 pacientes recibieron raquianestesia simple (Grupo 1), 100 recibieron bloqueo combinado raqui-peridural (Grupo 2) y 100 recibieron raquianestesia continua (Grupo 3) en los últimos 


\section{IMBELLONI AND BEATO}

cuatro años. Todos los bloqueos fueron realizados en decúbito lateral izquierdo. Fueron evaluados: suceso de punción, nivel de analgesia, bloqueo motor de miembros inferiores, calidad de la anestesia, necesidad de complementación, incidencia de fallas, parestesias, cefalea pós-punción, alteraciones cardiovasculares, confusión mental y delirium, transfusión sanguínea y mortalidad.

Resultados - No existe diferencia significativa entre los grupos en relación a la edad, peso y sexo. Los pacientes del grupo 2 fueron menores de que los del grupo 1 y 3. Las dosis utilizadas fueron de 15,30 mg de bupivacaína en el grupo 1; 23,68 mg en el grupo 2 y 10,10 $\mathrm{mg}$ en el grupo 3. No fue encontrada diferencia significativa $(p<0,01)$ entre los grupos, siendo menor con la raquianestesia continua y mayor con el BCRP. Existe una diferencia significativa $(p<0,01)$ en la dispersión cefálica entre los grupos 1 y 2, 1 y 3 y 2 y 3 , siendo menor con la raquianestesia continua y mayor con el BCRP. Todos los pacientes presentaron bloqueo motor completo. No existe diferencia significativa en relación a hipotensión arterial, bradicardia, parestesia y necesidad de sangre. Diecinueve pacientes presentaron confusión mental en el pós-operatorio, sin diferencia entre las técnicas utilizadas. No hay diferencia significativa en la incidencia de óbitos en la primera semana y en el primero mes de pós-operatorio.

Conclusiones - Los estudios retrospectivos generalmente cuestan poco y necesitan de menor tiempo cuando comparado con estudios controlados. Este estudio retrospectivo muestra que las técnicas de anestesia regional cursan con una baja incidencia de mortalidad en el primero mes y baja incidencia de complicaciones. 